\title{
Well-balanced computations of weak local residuals for the shallow water equations
}

Sudi Mungkasi ${ }^{1} \quad$ Stephen G. Roberts ${ }^{2}$

(Received 2 March 2015; revised 30 November 2015)

\begin{abstract}
The one-dimensional shallow water equations describe mass conservation and momentum conservation. We propose a well-balanced numerical technique for computing weak local residuals of the momentum equation. We compare the performance of weak local residuals of the momentum equation to those of the mass equation. All weak local residuals behave similarly.
\end{abstract}

\section{Contents}

1 Introduction

C129

http://journal.austms.org.au/ojs/index.php/ANZIAMJ/article/view/9369 gives this article, (C) Austral. Mathematical Soc. 2015. Published December 9, 2015, as part of the Proceedings of the 17th Biennial Computational Techniques and Applications Conference. ISSN 1446-8735. (Print two pages per sheet of paper.) Copies of this article must not be made otherwise available on the internet; instead link directly to this URL for this article. 
2 Weak local residuals for balance laws

C130

3 Shallow water equations

C133

3.1 Well-balancing the weak local residual . . . . . . . . C134

3.2 Wet/dry interface treatment for the weak local residual . . C136

4 Numerical tests

C137

4.1 Well-balanced test . . . . . . . . . . . . C C137

4.2 Dam break test involving a dry area and moving shock . . C141

4.3 Flow involving a stationary shock on an obstruction . . . . C141

5 Conclusions

C143

References

C146

\section{Introduction}

Karni, Kurganov and Petrova [5] originally proposed using weak local residuals (or local truncation errors) as smoothness indicators for conservation laws. The available theory supporting weak local residuals as smoothness indicators is only valid for scalar conservation laws, but with numerical experiments weak local residuals as smoothness indicators were seen to be also valid for systems of conservation laws $[4,5]$. A conservation law is homogeneous and so does not have any source terms. Section 2 derives the weak local residual for the scalar balance law.

In this article we measure the smoothness of solutions of the one-dimensional shallow water equations. The shallow water equations form a system of balance laws, that is, a system of conservation laws with an additional nonzero source term. The system consists of a homogeneous equation of mass conservation and a non-homogeneous equation of momentum conservation. We propose a numerical technique for measuring the smoothness of momentum by computing the weak local residuals of the momentum equation. Mungkasi 
and Roberts [9] discussed measuring the smoothness of water height using mass conservation. In Section 3 we describe our numerical technique for computing the weak local residuals of the momentum equation.

Due to the existence of a source term in the momentum equation (equation of momentum conservation), the computation of the equation's weak local residuals must be well-balanced. A non-well-balanced computation of a weak local residual may lead to spurious oscillations of the residual, leading to incorrect smoothness or error indicators. In this article we consider the steady state of a lake at rest. We develop well-balanced treatments for two different weak local residuals of the momentum equation. In addition, a wet/ dry interface treatment is also given in order to maintain the well-balanced property at wet/dry interfaces. Section 4 provides numerical tests for the performance of the weak local residuals.

\section{Weak local residuals for balance laws}

Kurganov et al. $[4,5]$ formulated the weak local residuals for conservation laws. These conservation laws are homogeneous, that is, they do not have source terms. We extend the work of Kurganov et al. [4, 5] to formulations of the weak local residuals of balanced laws. Balanced laws are conservation laws with additional nonzero source terms.

Consider the scalar balance law

$$
\begin{cases}q_{t}+f(q)_{x}=s, & -\infty<x<\infty, \\ q(x, t)=q_{0}(x), & t=0 .\end{cases}
$$

Here $x$ is a one-dimensional space variable, $t$ is the time variable, $q$ is the concentration of a quantity of interest, $f$ is the flux of $q, s$ is a source function and $q_{0}$ is an arbitrary function which defines the initial condition of $q$. Here $q$ and $s$ are both functions of $x$ and $t$. The weak form of the initial value 
problem (1) is

$$
\begin{aligned}
& \int_{0}^{\infty} \int_{-\infty}^{\infty}\left[q(x, t) T_{t}(x, t)+f(q(x, t)) T_{x}(x, t)+s(x, t) T(x, t)\right] d x d t \\
& +\int_{-\infty}^{\infty} q_{0}(x) T(x, 0) d x=0,
\end{aligned}
$$

where $T(x, t)$ is an arbitrary test function. A mathematical derivation of this weak form was derived by Knobel [6] and Smoller [12].

Given a fixed spatial step $\Delta x$ and temporal step $\Delta t$, we form a uniform spatial/ temporal grid made up of discrete spatial points $x_{j}:=j \Delta x$ for $j=0,1, \ldots, M$ and temporal points $t^{n}:=n \Delta t$ for $n=0,1, \ldots, N$. We denote $q_{j}^{n}$ as the approximate value of $q\left(x_{j}, t^{n}\right)$ computed by a conservative method. Like Karni and Kurganov [4], we denote the corresponding piecewise constant approximation as

$$
\mathrm{q}^{\Delta}(x, \mathrm{t}):=\mathrm{q}_{j}^{\mathrm{n}} \quad \text { if } \quad(x, \mathrm{t}) \in\left[\mathrm{x}_{j-1 / 2}, \mathrm{x}_{\mathrm{j}+1 / 2}\right] \times\left[\mathrm{t}^{\mathrm{n}-1 / 2}, \mathrm{t}^{\mathrm{n}+1 / 2}\right],
$$

where $x_{j \pm 1 / 2}:=x_{j} \pm \Delta x / 2$ and $t^{n \pm 1 / 2}:=t^{n} \pm \Delta t / 2$. We construct a test function $T_{j}^{n}(x, t):=B_{j}(x) B^{n}(t)$, where $B_{j}(x)$ and $B^{n}(t)$ are quadratic $B-$ splines centered at $x=x_{j}$ and $t=t^{n}$ with supports of size $3 \Delta x$ and $3 \Delta t$. That is,

$$
B_{j}(x)= \begin{cases}\frac{1}{2}\left(\frac{x-x_{j-3 / 2}}{\Delta x}\right)^{2} & \text { if } x_{j-3 / 2} \leqslant x \leqslant x_{j-1 / 2} \\ \frac{3}{4}-\left(\frac{x-x_{j}}{\Delta x}\right)^{2} & \text { if } x_{j-1 / 2} \leqslant x \leqslant x_{j+1 / 2} \\ \frac{1}{2}\left(\frac{x-x_{j+3 / 2}}{\Delta x}\right)^{2} & \text { if } x_{j+1 / 2} \leqslant x \leqslant x_{j+3 / 2} \\ 0 & \text { otherwise }\end{cases}
$$

and

$$
B^{n}(t)= \begin{cases}\frac{1}{2}\left(\frac{t-t^{n-3 / 2}}{\Delta t}\right)^{2} & \text { if } t^{n-3 / 2} \leqslant t \leqslant t^{n-1 / 2} \\ \frac{3}{4}-\left(\frac{t-t^{n}}{\Delta t}\right)^{2} & \text { if } t^{n-1 / 2} \leqslant t \leqslant t^{n+1 / 2} \\ \frac{1}{2}\left(\frac{t-t^{n+3 / 2}}{\Delta t}\right)^{2} & \text { if } t^{n+1 / 2} \leqslant t \leqslant t^{n+3 / 2} \\ 0 & \text { otherwise. }\end{cases}
$$


Then substituting the test function $T_{j}^{n}(x, t)$ into (2) leads to a weak form of the local residual $[4,5]$ for the balance law

$$
\begin{aligned}
E_{j}^{n}= & -\int_{t^{n-3 / 2}}^{t^{n+3 / 2}} \int_{x_{j-3 / 2}}^{x_{j+3 / 2}}\left\{q^{\Delta}(x, t)\left[T_{j}^{n}(x, t)\right]_{t}\right. \\
& \left.+f\left(q^{\Delta}(x, t)\right)\left[T_{j}^{n}(x, t)\right]_{x}+s^{\Delta}(x, t) T_{j}^{n}(x, t)\right\} d x d t .
\end{aligned}
$$

The weak local residual (6) is then

$$
\begin{aligned}
E_{j}^{n}= & \frac{\Delta x}{12}\left[q_{j+1}^{n+1}-q_{j+1}^{n-1}+4\left(q_{j}^{n+1}-q_{j}^{n-1}\right)+q_{j-1}^{n+1}-q_{j-1}^{n-1}\right] \\
& +\frac{\Delta t}{12}\left[f\left(q_{j+1}^{n+1}\right)-f\left(q_{j-1}^{n+1}\right)+4\left(f\left(q_{j+1}^{n}\right)-f\left(q_{j-1}^{n}\right)\right)\right. \\
& \left.+f\left(q_{j+1}^{n-1}\right)-f\left(q_{j-1}^{n-1}\right)\right] \\
& -\frac{\Delta x \Delta t}{36}\left[\left(s_{j-1}^{n-1}+4 s_{j-1}^{n}+s_{j-1}^{n+1}\right)+4\left(s_{j}^{n-1}+4 s_{j}^{n}+s_{j}^{n+1}\right)\right. \\
& \left.+\left(s_{j+1}^{n-1}+4 s_{j+1}^{n}+s_{j+1}^{n+1}\right)\right] .
\end{aligned}
$$

Using quadratic B-splines in constructing the test function is an adaption of the work of Karni, Kurganov, and Petrova [5] on conservation laws. So we refer to the weak local residual (7) as KKP (Karni-Kurganov-Petrova).

Rather than quadratic B-splines, we can also choose localized linear B-splines as the test functions $T_{j+1 / 2}^{n-1 / 2}(x, t):=B_{j+1 / 2}(x) B^{n-1 / 2}(t)$, where $B_{j+1 / 2}(x)$ and $B^{n-1 / 2}(t)$ are centered at $x=x_{j+1 / 2}$ and $t=t^{n-1 / 2}$ with supports of size $2 \Delta x$ and $2 \Delta t$. That is,

$$
B_{j+1 / 2}(x)= \begin{cases}\frac{x-x_{j-1 / 2}}{\Delta x} & \text { if } x_{j-1 / 2} \leqslant x \leqslant x_{j+1 / 2} \\ \frac{x_{j+3 / 2}-x}{\Delta x} & \text { if } x_{j+1 / 2} \leqslant x \leqslant x_{j+3 / 2} \\ 0 & \text { otherwise }\end{cases}
$$

and

$$
B^{n-1 / 2}(t)= \begin{cases}\frac{t-t^{n-3 / 2}}{\Delta t} & \text { if } t^{n-3 / 2} \leqslant t \leqslant t^{n-1 / 2} \\ \frac{t^{n+1 / 2}-t}{\Delta t} & \text { if } t^{n-1 / 2} \leqslant t \leqslant t^{n+1 / 2} \\ 0 & \text { otherwise. }\end{cases}
$$


This results in a less expensive computation of the weak local residual

$$
\begin{aligned}
E_{j+1 / 2}^{n-1 / 2}= & -\int_{t^{n-3 / 2}}^{t^{n+1 / 2}} \int_{x_{j-1 / 2}}^{x_{j+3 / 2}}\left\{q^{\Delta}(x, t)\left[T_{j+1 / 2}^{n-1 / 2}\right]_{t}+f\left(q^{\Delta}(x, t)\right)\left[T_{j+1 / 2}^{n-1 / 2}\right]_{x}\right. \\
& \left.+s^{\Delta}(x, t) T_{j+1 / 2}^{n-1 / 2}\right\} d x d t,
\end{aligned}
$$

which after a straightforward computation becomes

$$
\begin{aligned}
E_{j+1 / 2}^{n-1 / 2}= & \frac{\Delta x}{2}\left[q_{j}^{n}-q_{j}^{n-1}+q_{j+1}^{n}-q_{j+1}^{n-1}\right] \\
& +\frac{\Delta t}{2}\left[f\left(q_{j+1}^{n-1}\right)-f\left(q_{j}^{n-1}\right)+f\left(q_{j+1}^{n}\right)-f\left(q_{j}^{n}\right)\right] \\
& -\frac{\Delta x \Delta t}{4}\left[s_{j}^{n-1}+s_{j}^{n}+s_{j+1}^{n-1}+s_{j+1}^{n}\right] .
\end{aligned}
$$

Using linear B-splines to construct the test function is an adaption of the work of Constantin and Kurganov [3] on conservation laws. So we refer to the weak local residual (11) as CK (Constantin-Kurganov).

\section{Shallow water equations}

The shallow water equations are

$$
\begin{aligned}
h_{\mathrm{t}}+(h \mathrm{u})_{\mathrm{x}} & =0, \\
(h \mathrm{u})_{\mathrm{t}}+\left(h \mathrm{u}^{2}+\frac{1}{2} g \mathrm{~h}^{2}\right)_{\mathrm{x}} & =-g h z_{x} .
\end{aligned}
$$

Here, $x$ is the coordinate in one-dimensional space, $t$ is time, $u(x, t)$ denotes the water velocity, $h(x, t)$ denotes the water height, $z(x)$ is the topography, and $\mathrm{g}$ is the acceleration due to gravity. Another quantity of interest is the stage $w=w(x, t)$ which is the water surface elevation given by $w=h+z$.

The non-homogeneous shallow water equations (12)-(13) are a system of balance laws. Implementing KKP (7) and/or CK (11) naïvely as smoothness 
indicators for balance laws may lead to spurious oscillations of the indicator values. We show an example of this problem in Section 4. The problem is caused by source terms in the balance laws. To make the indicators work properly, we need to make the computation of the indicators well-balanced.

Mungkasi and Roberts [9] presented weak local residuals of the mass equation (equation of mass conservation) as smoothness indicators. No well-balanced technique was needed in that case since the mass equation does not have a source term. In the next two subsections we focus on well-balancing the weak local residual of the non-homogeneous momentum equation. We limit our discussion on the well-balanced technique to the steady state of a lake at rest.

\subsection{Well-balancing the weak local residual}

We propose a well-balanced technique for computing weak local residuals of the non-homogeneous momentum equation (13) in wet regions.

Consider the non-homogeneous momentum equation (13) and the weak local residual (7). For the steady state of a lake at rest, the weak local residual (7) simplifies to

$$
\begin{aligned}
E_{j}^{n}= & \frac{\Delta t}{12}\left[f\left(q_{j+1}^{n+1}\right)-f\left(q_{j-1}^{n+1}\right)\right]-\frac{\Delta x \Delta t}{36}\left[s_{j+1}^{n+1}+4 s_{j}^{n+1}+s_{j-1}^{n+1}\right] \\
& +\frac{\Delta t}{3}\left[f\left(q_{j+1}^{n}\right)-f\left(q_{j-1}^{n}\right)\right]-\frac{\Delta x \Delta t}{9}\left[s_{j+1}^{n}+4 s_{j}^{n}+s_{j-1}^{n}\right] \\
& +\frac{\Delta t}{12}\left[f\left(q_{j+1}^{n-1}\right)-f\left(q_{j-1}^{n-1}\right)\right]-\frac{\Delta x \Delta t}{36}\left[s_{j+1}^{n-1}+4 s_{j}^{n-1}+s_{j-1}^{n-1}\right] .
\end{aligned}
$$

In the case of a still lake $f\left(q_{a}^{b}\right)=\frac{1}{2} g\left(h_{a}^{b}\right)^{2}$. For our indicator to be wellbalanced for the still lake case we need

$$
\left[f\left(q_{j+1}\right)-f\left(q_{j-1}\right)\right]=\frac{\Delta x}{3}\left[s_{j+1}+4 s_{j}+s_{j-1}\right],
$$

for each timestep $n-1, n$ and $n+1$ (we drop the timestep super-scripts). 
Table 1: Discrete values for the source terms in KKP and CK.

\begin{tabular}{ll|ll} 
KKP terms & Discrete values & CK terms & Discrete values \\
\hline$s_{j-1}$ & $-g h_{j-1} \frac{z_{j+1}-z_{j-1}}{2 \Delta x}$ & $s_{j}$ & $-g h_{j} \frac{z_{j+1}-z_{j}}{\Delta x}$ \\
$s_{j}$ & $-g \frac{h_{j+1}+h_{j-1} \frac{z_{j+1}-z_{j-1}}{2}}{2}$ & $s_{j+1}$ & $-g h_{j+1} \frac{z_{j+1}-z_{j}}{\Delta x}$ \\
$s_{j+1}$ & $-g h_{j+1} \frac{z_{j+1}-z_{j-1}}{2 \Delta x}$ & &
\end{tabular}

Since the fluxes in $(15)$ are defined at the $(j-1)$ th and $(j+1)$ th cells, we need to enforce that the source term discretisations involve only the $(j-1)$ th and $(j+1)$ th cells. That is, we take $s_{j}=\frac{1}{2}\left(s_{j+1}+s_{j-1}\right)$. Equation (15) is then rewritten as

$$
\frac{g}{2}\left[\left(h_{j+1}\right)^{2}-\left(h_{j-1}\right)^{2}\right]=\Delta x\left[s_{j+1}+s_{j-1}\right] .
$$

Next, we use the same value for the topography gradient $z_{x}$ in the formulations of $s_{j+1}$ and $s_{j-1}$, so that $h_{j+1}-h_{j-1}=z_{j-1}-z_{j+1}$ for the lake at rest. This is achieved by discretising

$$
\left(z_{x}\right)_{j+1}=\left(z_{x}\right)_{j-1}:=\left(z_{j+1}-z_{j-1}\right) /(2 \Delta x) .
$$

This last enforcement guarantees the well-balanced computation of the weak local residual (7). This source term discretisation technique is adapted from numerical schemes originally proposed by Bermudez and Vazquez [2]. This technique was also used by Audusse et al. [1] and Noelle et al. [10] to develop well-balanced numerical schemes. Each source term in KKP (7) and its discretisation using the above technique is given in the first and second column of Table 1, respectively.

Using a similar technique as described above, each source term in CK (11) and its discretisation are given in the third and fourth column of Table 1, respectively. 


\subsection{Wet/dry interface treatment for the weak local residual}

The well-balanced technique describe in Section 3.1 is valid for all-wet regions, that is, the $(j-1)$ th, $j$ th, and $(j+1)$ th cells are wet. A numerical treatment at a wet/dry interface is needed and we describe it here. We omit the description of a dry/wet treatment, as it is similar to the treatment of a wet/dry interface.

We solve the shallow water equations using the finite volume method described by Mungkasi and Roberts [8]. We implement the discretisation proposed by Audusse et al. [1]. Quantities are reconstructed based on height $h$, stage $w:=$ $h+z$, and velocity $u$. We implement the wet/dry interface reconstruction, proposed by Audusse et al. [1]. The wet/dry interface reconstruction adjusts the bed topography $z$ if negative water height occurs, so that the water height $h$ remains nonnegative $[1,8$, for more details]. A second order method with a minmod limiter is used [11].

Since the KKP indicator of the $j$ th cell involves three cells, namely the $(j-1)$ th, $j$ th, and $(j+1)$ th cells, there are two cases of the wet/dry interface for the KKP indicator: (a) wet-dry-dry, that is, wet for the $(j-1)$ th cell, dry for the jth cell, dry for the $(j+1)$ th cell; and (b) wet-wet-dry, that is, wet for the $(j-1)$ th cell, wet for the $j$ th cell, dry for the $(j+1)$ th cell. These two cases are illustrated in Figure 1. In the computation of the KKP indicator for these cases, $z_{j+1}$ is replaced by $w_{j-1}$. This ensures that the indicator computation at the wet/dry interface remains well-balanced. The two remaining special cases, namely wet-dry-wet and dry-wet-dry, are assumed to have indicator values of zero.

The treatment of a wet/dry interface computation of the CK indicator is analogous to the KKP computation. Since the CK indicator considers only two cells, namely the $j$ th and $(j+1)$ th cells, for computing the weak local residual, there is only one case of wet/dry interface. That is, the jth cell is wet but the $(j+1)$ th cell is dry. In this case $z_{j+1}$ is replaced by $w_{j}$, so that that the indicator computation at the wet/dry interface remains well-balanced. 
Figure 1: Two cases of the wet/dry interface for the KKP indicator: (a) the wetdry-dry case; (b) the wet-wet-dry case. The dashed horizontal line indicates the water level.

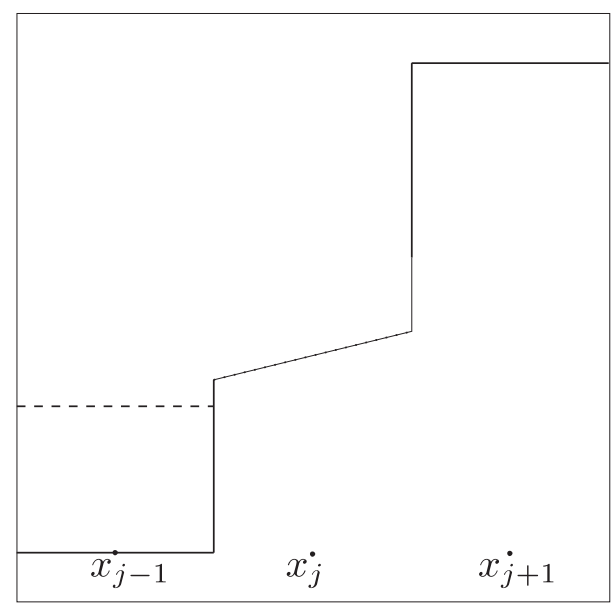

(a)

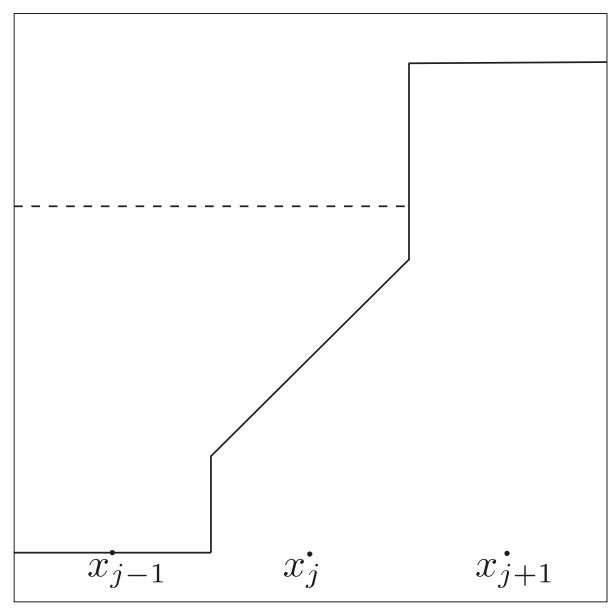

(b)

\section{$4 \quad$ Numerical tests}

We test the performance of weak local residuals. We use the second order well-balanced finite volume method [8]. Quantities are measured in SI units.

\subsection{Well-balanced test}

Consider a lake at rest with a discontinuous island in the middle of the lake, illustrated in Figure 2. This test is an adaptation of the test of Mungkasi and Roberts [8]. In the computation, the spatial domain is discretised into 800 cells.

At time $t=2$, four indicators, namely $\mathrm{CK}_{-} \mathrm{H}, \mathrm{CK} \_\mathrm{UH}, \mathrm{KKP} \_$Hand $\mathrm{KKP} \_\mathrm{UH}$, are depicted in Figure 3. Indicators $\mathrm{CK}_{-}{ }_{\mathrm{H}}$ and $\overline{\mathrm{CK}} \_\mathrm{UH}$ are the weak local 
Figure 2: A cross-section of a lake at rest with a discontinuous island in the middle [8].

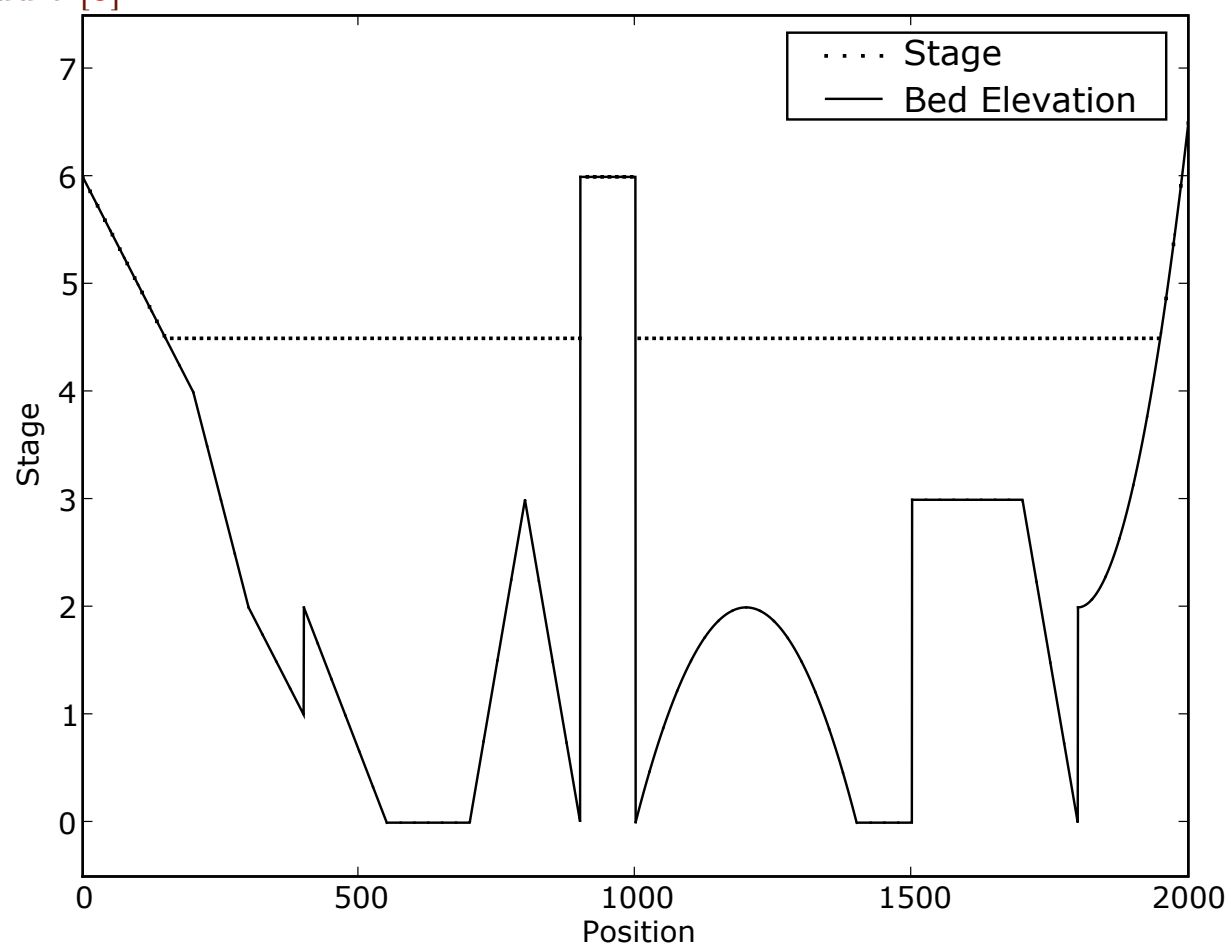

residuals of the mass and momentum equations based on (11), while KKP_H and KKP_UH are the weak local residuals of the mass and momentum equations based on (7). All indicators are the correct weak local residuals of the steady state of a lake at rest, correct to the order of $10^{-15}$ (machine precision).

In contrast, non-well-balanced computations of weak local residuals could lead to incorrect behaviour of the residuals, as unphysical oscillations may occur. Figure 4 shows this incorrect behaviour of weak local residuals. In this Figure 4, CK_UH and KKP_UH are shown without scaling and computed naïvely using local cell quantity values found from finite volume quantity 
Figure 3: The weak local residuals at time $t=2$. The four vertical axis scales are $10^{16} \times \mathrm{CK} \_\mathrm{H}, 10^{15} \times \mathrm{CK} \_\mathrm{UH}, 10^{16} \times \mathrm{KKP} \_\mathrm{H}$ and $10^{15} \times \mathrm{KKP} \_\mathrm{UH}$, respectively.

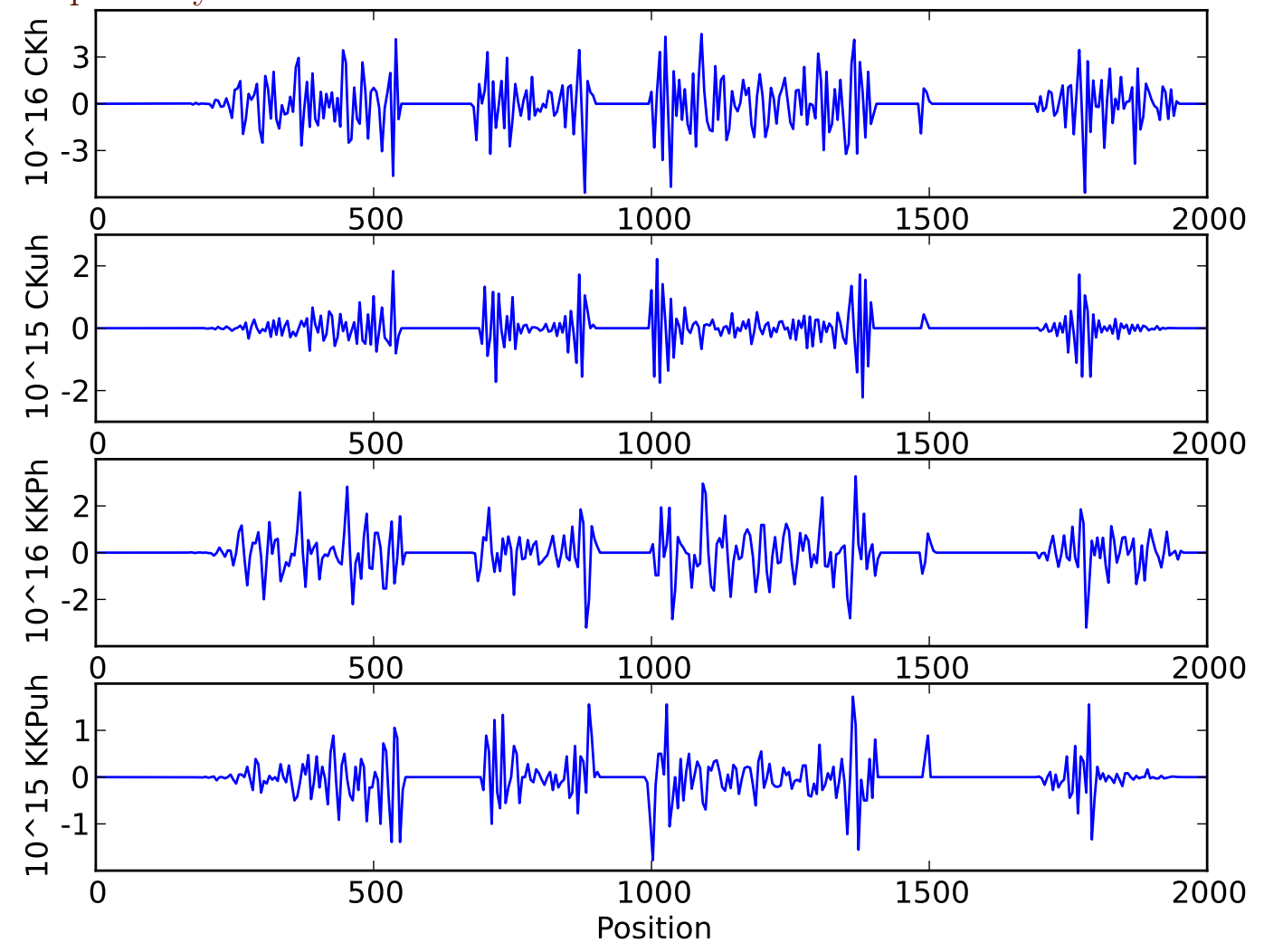


Figure 4: Unphysical weak local residuals at time $t=2$. Here CK_UH and KKP_UH are computed naïvely using local cell quantity values based on finite volume quantity reconstructions. Both CK_UH and KKP_UH are shown without scaling.
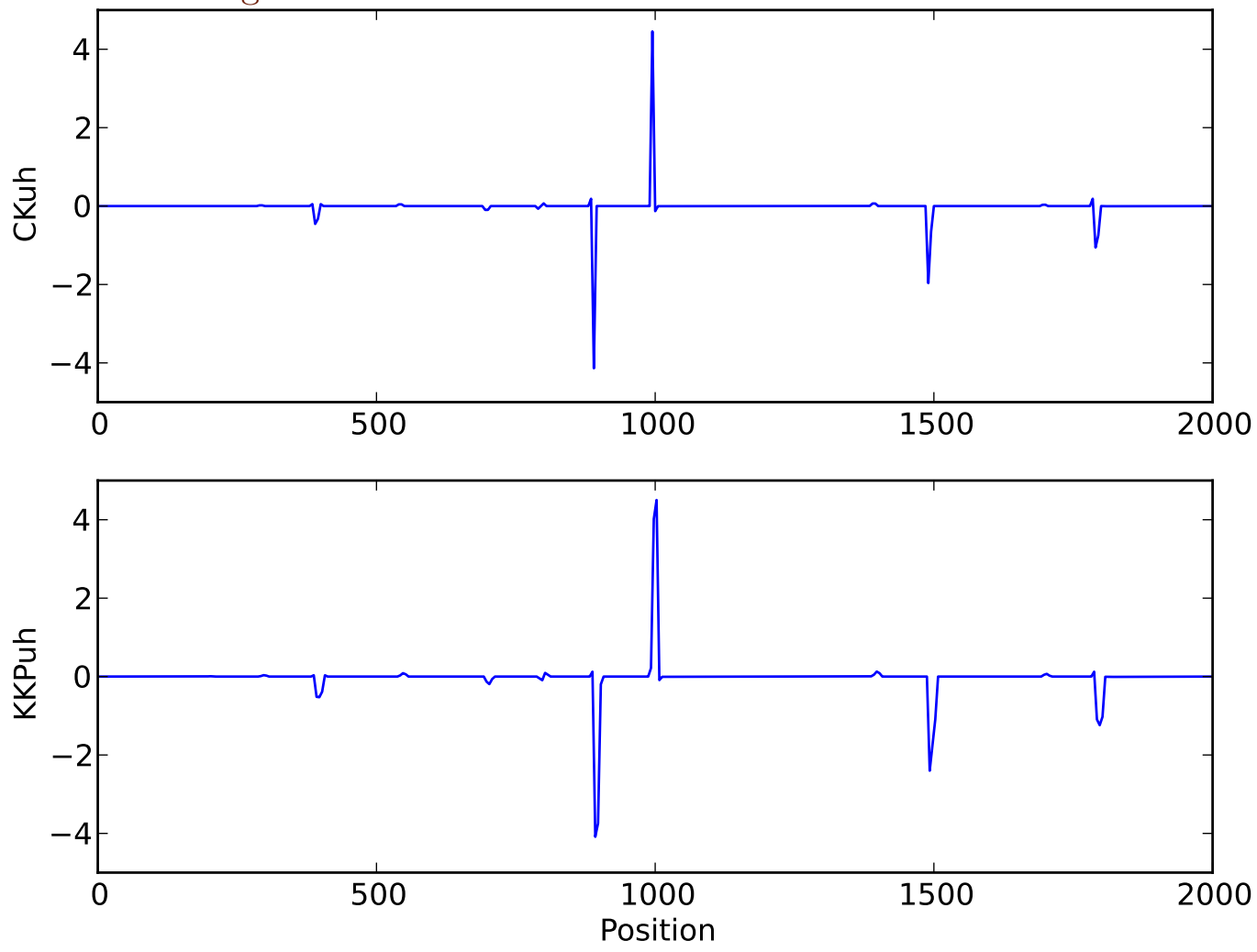
reconstructions. In this case, $\mathrm{CK}_{-} \mathrm{UH}_{\mathrm{H}}$ and $\mathrm{KKP} \_\mathrm{UH}$ have large indicator values at some points, although these values should actually be zero.

\subsection{Dam break test involving a dry area and moving shock}

Consider the collapse of a reservoir on a horizontal topography [9]

$$
z(x)=0, \quad 0<x<2000
$$

with initial velocity and stage

$$
u(x, 0)=0, \quad w(x, 0)= \begin{cases}0 & \text { if } 0<x<500 \\ 10 & \text { if } 500<x<1500 \\ 5 & \text { if } 1500<x<2000\end{cases}
$$

The simulation illustrates the motion of the water at any point $x$ in the domain and at any time $t>0$ with respect to the initial condition (19). Condition (19) describes two dam walls (at $x=500$ and $x=1500$ ). In the computation, the spatial domain is discretised into 800 cells.

The simulation results at time $t=20$ are shown in Figures 5 and 6 . Figure 5 shows the stage and the corresponding momentum value. The weak local residuals $\mathrm{CK}_{-} \mathrm{H}_{2} \mathrm{CK}_{-} \mathrm{UH}, \mathrm{KKP} \_\mathrm{H}$, and $\mathrm{KKP} \_\mathrm{UH}$ are shown in Figure 6. The $\mathrm{CK}_{-} \mathrm{H}$ indicator is best at identifying the shock.

\subsection{Flow involving a stationary shock on an obstruction}

Consider a channel of length 25 with a parabolic bump topography [9]

$$
z(x)= \begin{cases}0.2-0.05(x-10)^{2} & \text { if } 8 \leqslant x \leqslant 12 \\ 0 & \text { otherwise }\end{cases}
$$


Figure 5: The dam break stage and momentum at time $t=20$.
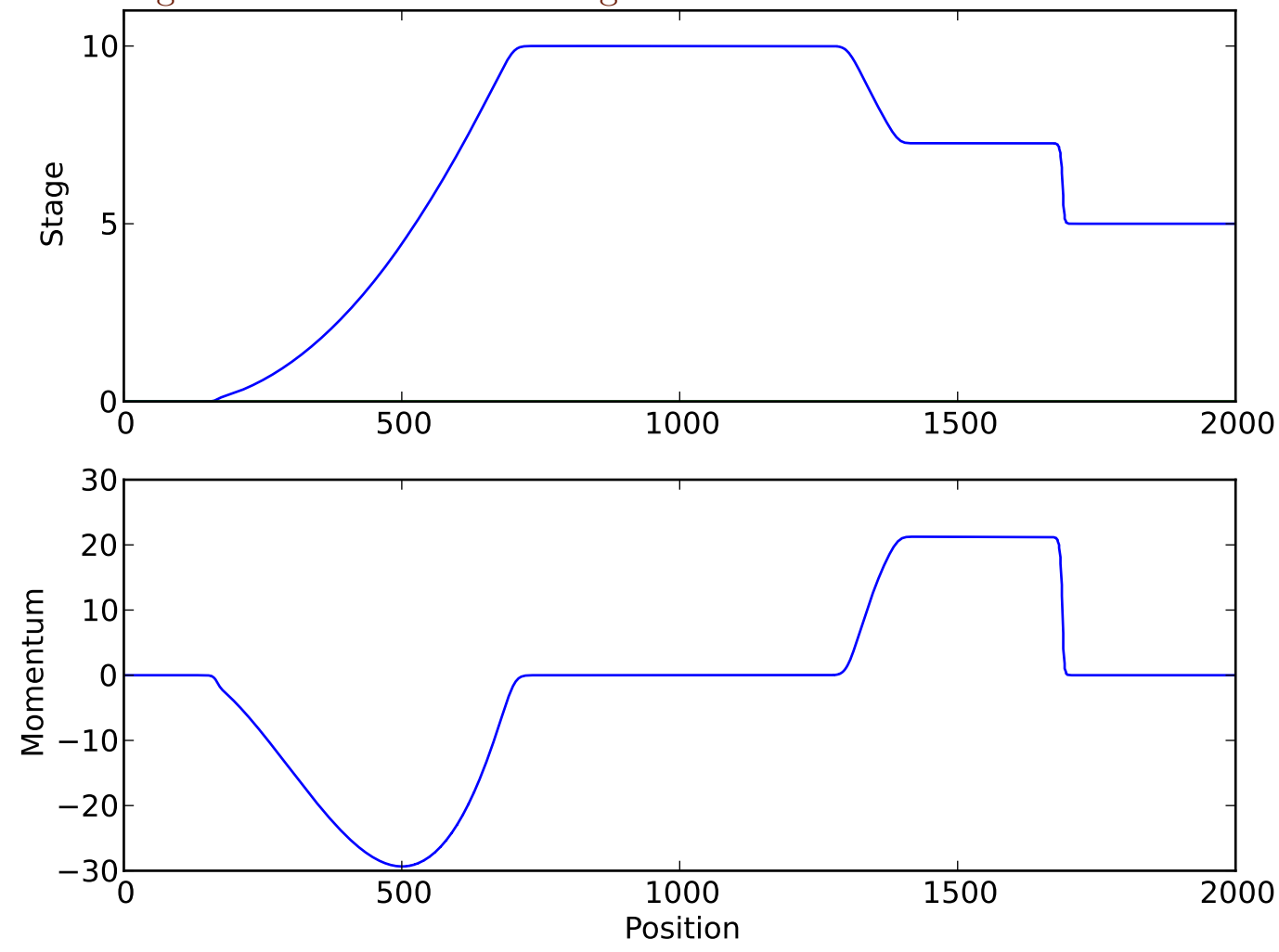

and an initial condition

$$
u(x, 0)=0, \quad w(x, 0)=0.33
$$

together with the Dirichlet boundary conditions

$$
\begin{aligned}
& {[w, h u, z, h, u]=[0.42,0.18,0.0,0.42,0.18 / 0.42] \quad \text { at } x=0^{-} \text {, }} \\
& {[w, h u, z, h, u]=[0.33,0.18,0.0,0.33,0.18 / 0.33] \quad \text { at } x=25^{+} \text {. }}
\end{aligned}
$$

In the computation, the spatial domain is discretised into 400 cells.

The simulation results at time $t=100$ are shown in Figures 7 and 8 . Figure 7 shows the stage and the corresponding momentum. The weak local residuals 
Figure 6: The dam break weak local residuals at time $t=20$.
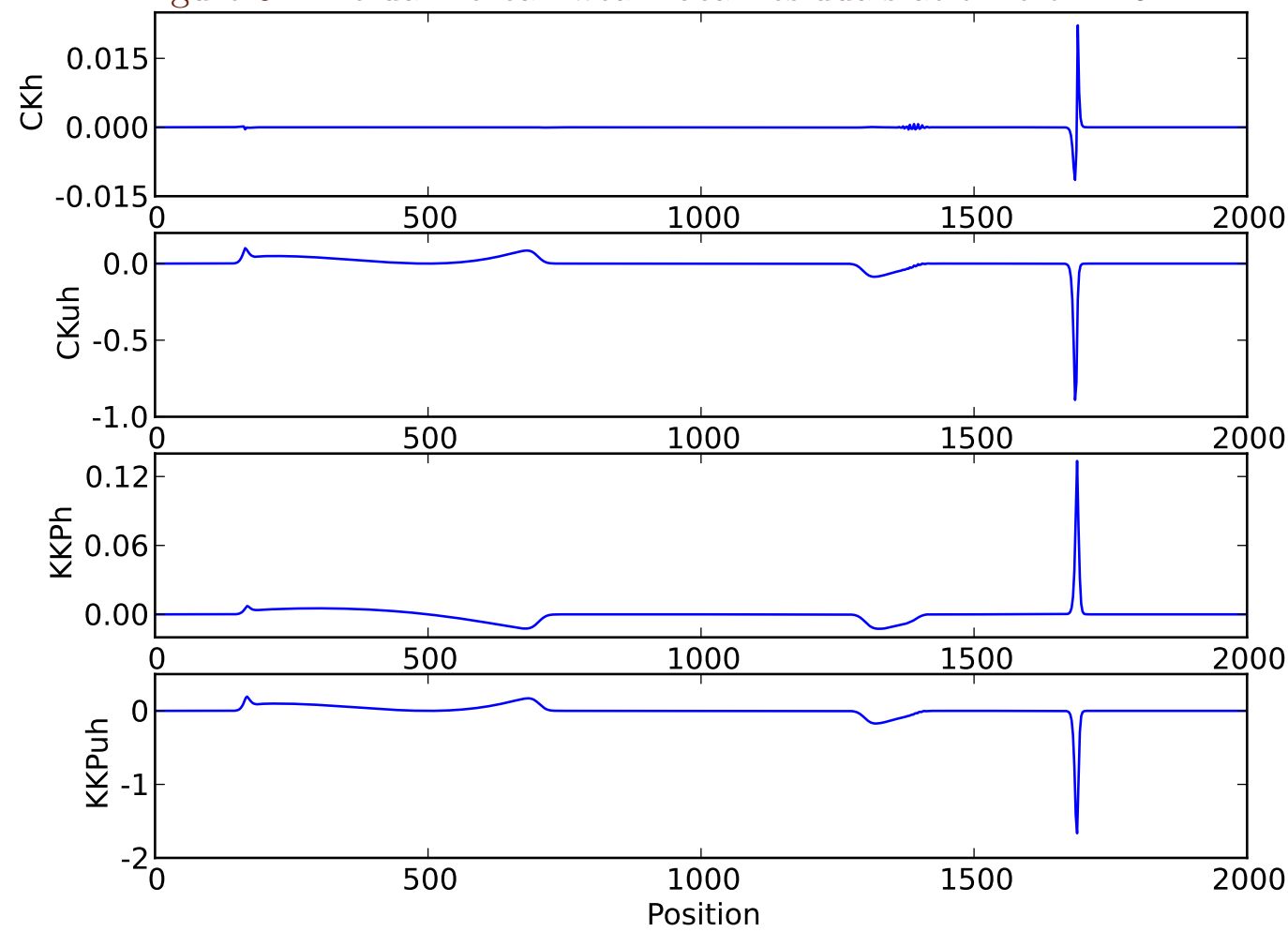

CK_H, CK_UH, KKP_H, and KKP_UH are shown in Figure 8. More details of these results are given by Mungkasi [7].

\section{Conclusions}

Weak local residuals were implemented to measure the smoothness of solutions of the shallow water equations. Even though the theory of weak local residuals as smoothness indicators is, so far, only available for the scalar conservation law, weak local residuals can also be used as smoothness indicators for the 
Figure 7: The stage and momentum of steady flow over a parabolic obstruction at time $t=100$.
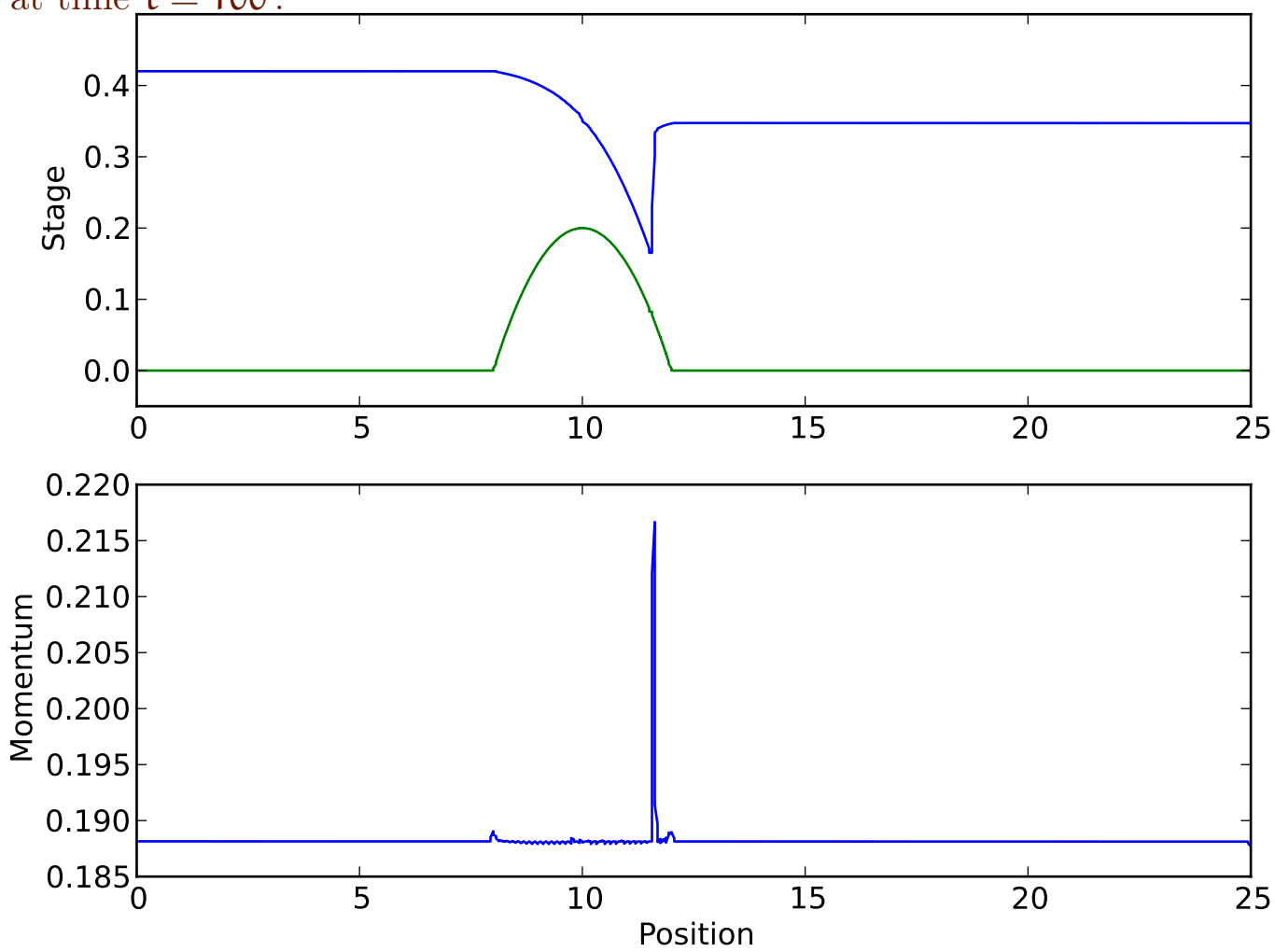

shallow water equations, which are a system of balance laws. This suggests that weak local residuals may also be used as smoothness indicators for a system of balance laws in general, as long as an appropriate well-balanced treatment is used.

Acknowledgements The work of Sudi Mungkasi was supported by ANU PhD and ANU Tuition Scholarships. 
Figure 8: The weak local residuals of steady flow over a parabolic obstruction at time $t=100$. The four vertical axis scales are $10^{5} \times \mathrm{CK}_{-} \mathrm{H}, 10^{5} \times \mathrm{CK} \_\mathrm{UH}$, $10^{5} \times \mathrm{KKP} \_\mathrm{H}$ and $10^{5} \times \mathrm{KKP} \_\mathrm{UH}$, respectively.
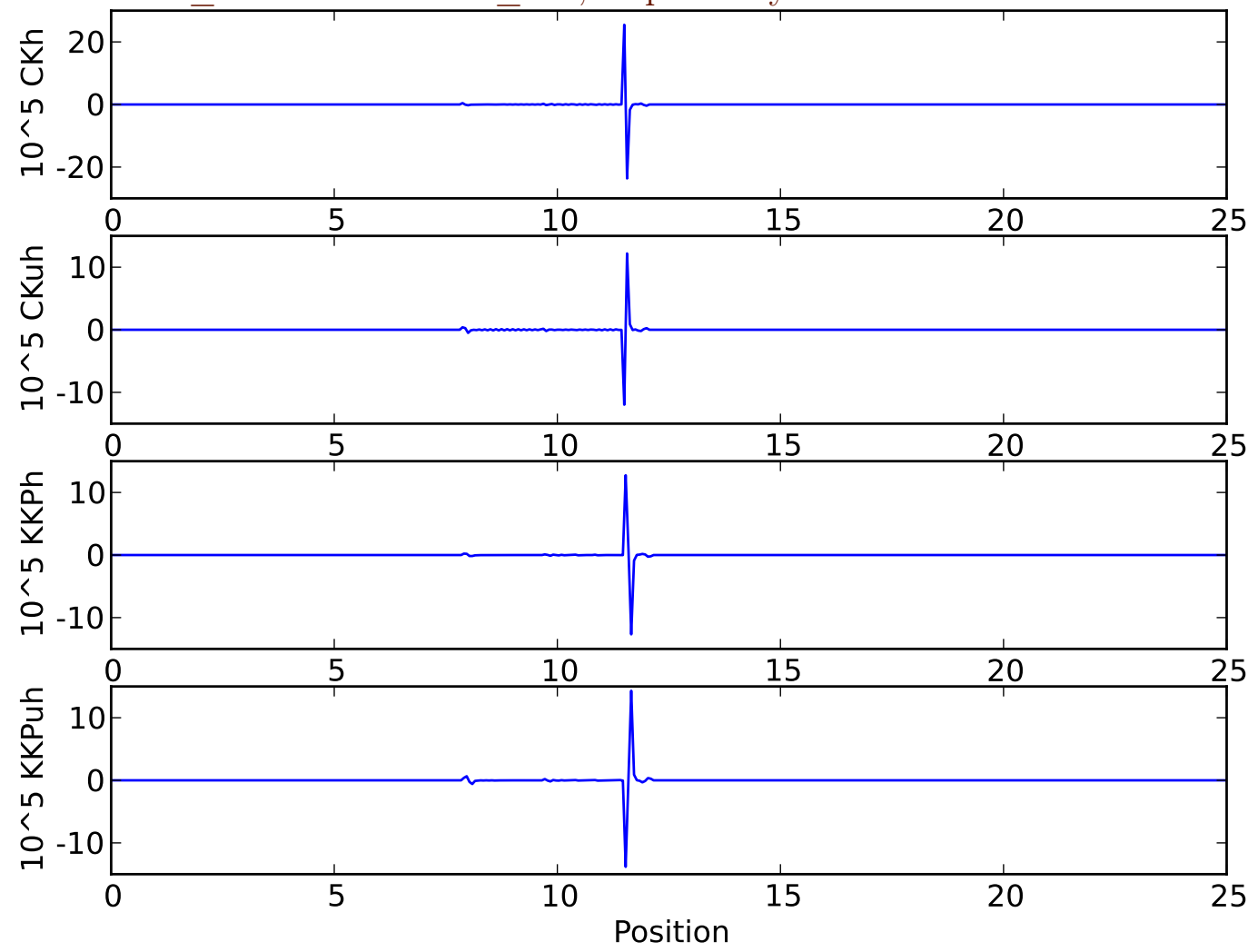


\section{References}

[1] E. Audusse, F. Bouchut, M. O. Bristeau, R. Klein, and B. Perthame. A fast and stable well-balanced scheme with hydrostatic reconstruction for shallow water flows. SIAM J. Sci. Comput. 25:2050-2065, 2004. doi:10.1137/S1064827503431090. C135, C136

[2] A. Bermudez and M. E. Vazquez. Upwind methods for hyperbolic conservation laws with source terms. Comput. Fluids, 23:1049-1071, 1994. doi:10.1016/0045-7930(94)90004-3. C135

[3] L. A. Constantin and A. Kurganov. Adaptive central-upwind schemes for hyperbolic systems of conservation laws. In F. Asakura, S. Kawashima, A. Matsumura, S. Nishibata, K. Nishihara (Eds.) Hyperbolic problems: Theory, numerics, and applications. Vol. 1 of Proceedings of the 10th international conference, Osaka, Japan, 13-17 September 2004, Yokohama Publishers, Yokohama, 2006, pages 95-103, 2006. http://www.ybook.co.jp/pub/ISBN4-946552-21-9.htm, http://129.81.170.14/ kurganov/Constantin-Kurganov.pdf C133

[4] S. Karni and A. Kurganov. Local error analysis for approximate solutions of hyperbolic conservation laws. Adv. Comput. Math. 22:79-99, 2005. doi:10.1007/s10444-005-7099-8 C129, C130, C131, C132

[5] S. Karni, A. Kurganov, and G. Petrova. A smoothness indicator for adaptive algorithms for hyperbolic systems. J. Comput. Phys. 178:323-341, 2002. doi:10.1006/jcph.2002.7024 C129, C130, C132

[6] R. Knobel. An Introduction to the Mathematical Theory of Waves. American Mathematical Society, Providence, 2000. http://www . ams . org/bookstore-getitem/item=stml-3 C131

[7] S. Mungkasi. A Study of Well-Balanced Finite Volume Methods and Refinement Indicators for the Shallow Water Equations. PhD thesis, 
Australian National University, Canberra, 2012.

http://hdl.handle.net/1885/10301 C143

[8] S. Mungkasi and S. G. Roberts. On the best quantity reconstructions for a well balanced finite volume method used to solve the shallow water wave equations with a wet/dry interface. ANZIAM J. 51:C48-C65, 2009 http://journal. austms.org.au/ojs/index.php/ANZIAMJ/article/ view/2576 C136, C137, C138

[9] S. Mungkasi and S. G. Roberts. Numerical entropy production for shallow water flows. ANZIAM J. 52:C1-C17, 2010. http://journal. austms.org.au/ojs/index.php/ANZIAMJ/article/view/3786 C130, C134, C141

[10] S. Noelle, N. Pankratz, G. Puppo, and J. R. Natvig. Well-balanced finite volume schemes of arbitrary order of accuracy for shallow water flows. $J$. Comput. Phy. 213:474-499, 2006. doi:10.1016/j.jcp.2005.08.019 C135

[11] P. L. Roe. Characteristic-based schemes for the Euler equations. Ann. Rev. Fluid Mech. 18:337-365, 1986. doi:10.1146/annurev.fl.18.010186.002005 C136

[12] J. Smoller. Shock Waves and Reaction-Diffusion Equations. Springer-Verlag, New York, 1983. doi:10.1007/978-1-4612-0873-0 C131

\section{Author addresses}

1. Sudi Mungkasi, Department of Mathematics, Sanata Dharma University, Yogyakarta, INDONESIA. mailto:sudi@usd.ac.id

2. Stephen G. Roberts, Mathematical Sciences Institute, Australian National University, Canberra, Australia. mailto:stephen.roberts@anu. edu.au 